13

\title{
Десять способов определения площади полевой эмиссии
}

\author{
() Е.О. Попов, А.Г. Колосько, М.А. Чумак, С.В. Филиппов \\ 1 Физико-технический институт им. А.Ф. Иоффре РАН, \\ 194021 Санкт-Петербург, Россия \\ e-mail: e.popov@mail.ioffe.ru
}

Поступило в Редакцию 29 декабря 2017 г.

В окончательной редакции 6 марта 2018 г.

Принято к публикации 16 апреля 2019 г.

Исследовано многообразие видов формального определения одного из основных параметров полевых эмиссионных систем - площади эмиссии. На примере модели „полусфера на цилиндрическом основании“, построенной с помощью программного пакета COMSOL Multiphysics, показано соотношение между известными видами оценок площади эмиссии. Рассчитаны соответствующие доли эмиссионных токов. Показано, что формальная площадь эмиссии (отношение полного тока к плотности тока на вершине) обеспечивает только $\sim 75 \%$ тока эмиссии. Получен эффект аномального поведения величины эффективной площади эмиссии (отсечка линии тренда вольт-амперных характеристик) при изменении уровня напряжения в стандартных координатах Фаулера-Нордгейма. Предложен способ экспериментальной оценки площади с применением модифицированных координат Фаулера-Нордгейма $\ln \left(I / U^{2-\eta / 6}\right)$ vs $1 / U$. Метод применен для анализа данных полевой эмиссии многоострийного нанокомпозитного эмиттера с углеродными нанотрубками.

Ключевые слова: полевая эмиссия, площадь полевой эмиссии, углеродные нанотрубки, моделирование электрических полей, уравнение Фаулера-Нордгейма.

DOI: 10.21883/JTF.2019.10.48182.2624

\section{Введение}

Полевая эмиссия - квантовый эффект перехода электронов через границу двух сред. Обнаруженный еще в девятнадцатом веке, он на сегодняшний день попрежнему недостаточно полно описан теоретиками и представляет достаточно серьезную проблему для анализа экспериментальных данных. Существенного улучшения эмиссионных свойств полевых катодов удалось достичь за счет использования наноструктурированных материалов (особенно на основе нанотрубок). Однако отсутствие четкого понимания эффектов, происходящих на эмиссионной поверхности, не позволяет создать образцы с характеристиками, достаточными для выведения изделий вакуумной наноэлектроники на широкий потребительский рынок [1-4]. Прототипы эмиссионных дисплеев, осветительных ламп, рентгеновских аппаратов и т. д. давно уже существуют в научных лабораториях, но нестабильность эмиссионных систем не может гарантировать их долговременную работу. Эта нестабильность, как правило, связана с флуктуациями тока и локальным перегревом отдельных эмиссионных центров.

Одним из ключевых факторов при переходе от математических зависимостей полевой эмиссии к реальным источникам электронов является площадь полевой эмиссии. Существуют различные методы экспериментального получения ее значений (по картинам свечения полевого эмиссионного проектора $[5,6]$, по изображениям сканирующего эмиссионного микроскопа SAFEM [7]), а также несколько способов ее определения в теоре- тических расчетах с применением как аналитических вычислений, так и компьютерного моделирования [8-10].

Важность определения данного параметра продиктована необходимостью сравнения свойств различных полевых катодов (чем выше площадь, способная к эмиссии, тем катод считается более стабильным). Кроме того, существует потребность аппроксимации экспериментальных вольт-амперных характеристик (BAX) катодов одним уравнением (как правило, это одна из вариаций уравнения Фаулера-Нордгейма), что позволяет охарактеризовать всю ВАХ несколькими эффективными параметрами и предсказать ее полный вид.

Целью настоящей работы было описание формализма и численное сравнение различных способов определения площади полевой эмиссии на примере результатов расчета эмиссионных токов одноострийного модельного катода. Катод представлял собой НРС (hemisphere-oncylindrical-post - полусфера на цилиндрическом основании) модель углеродной натроубки, построенную в программном пакете COMSOL. Расчеты величин площадей были выполнены специальной программой, написанной на языке LabView.

\section{Базовое уравнение потока электронов}

Плотность эмиссионного тока $j$ определяется величиной напряженности поля $F$ и зависит от формы потенциального барьера. Вывод формулы для треугольного барьера был впервые представлен в работе [11], а учет сил изображения в этом барьере (так называемый барьер 
Шоттки-Нордгейма) - в работе [12]. Более полная зависимость $j(F)$ с учетом влияния температуры была представлена в 1956 г. Мерфи и Гудом [13]. Для чистой полевой эмиссии (глубокое туннелирование) она имеет вид

$$
j=a_{F N} \varphi^{-1} \tau_{F}^{-2} F^{2} \exp \left(-b_{F N} \varphi^{3 / 2} v_{F} / F\right),
$$

где $a_{F N}$ и $b_{F N}-$ первая и вторая константы ФаулераНордгейма:

$$
\begin{gathered}
a_{F N}=e^{3} / 8 \pi h \approx 1.5 \cdot 10^{-6} \mathrm{~A} \cdot \mathrm{eV} \cdot \mathrm{V}^{2}, \\
b_{F N}=(8 \pi / 3 e h)\left(2 m_{e}\right)^{1 / 2} \approx 6.83 \cdot 10^{9} \mathrm{eV}^{-3 / 2} \cdot \mathrm{V} / \mathrm{m}
\end{gathered}
$$

а величины $\tau_{F}$ и $v_{F}$ являются специальными функциями, выраженными через эллиптические интегралы и слабо зависящими от поля $F$. Для определения этих функций можно использовать асимптотику, представленную в работе Форбса [14]:

$$
\begin{gathered}
\tau_{F}=1+f / 9-(f / 18) \ln f, \\
v_{F}=1-f+(f / 6) \ln f=s_{F}-u_{F} f,
\end{gathered}
$$

где $f$ - безразмерное поле на поверхности эмиттера: $f=F / F_{R}$, связанное с полем снятия потенциального барьера $F_{R}=\varphi^{2} 4 \pi \varepsilon_{0} / e^{3} \approx \varphi^{2} 694 \cdot 10^{8} \mathrm{~V} / \mathrm{m} / \mathrm{eV}^{2}$, а функции $s_{F}$ и $u_{F}$ зависят от этого поля: $s_{F}=1-f / 6$, $u_{F}=5 / 6-\ln (f / 6)$.

В результате подстановки уравнение (1) приобретает полубезразмерный вид

$$
\begin{aligned}
j= & a_{F N} \varphi^{-1}[1+f / 9-(f / 18) \ln f]^{-2} F^{2} \\
& \times \exp \left(-b_{F N} \varphi^{3 / 2}[1-f+(f / 6) \ln f] F^{-1}\right) .
\end{aligned}
$$

В некоторых случаях слабой зависимостью функций $\tau_{F}, v_{F}$ и $u_{F}$ от поля пренебрегают, подставляя вместо этих функций их значения при $f=0.3$ (середина диапазона значений напряженности поля, относящихся к режиму полевой эмиссии [15]): $\tau_{F}^{2} \approx 1.11$ и $v_{F} \approx 0.95-1.03 f$. Это преобразование приводит уравнение (1) к более простой зависимости

$$
\begin{aligned}
j= & 1.4 \cdot 10^{-6} \exp (10.17 / \sqrt{\varphi}) \varphi^{-1} F^{2} \\
& \times \exp \left(-6.49 \cdot 10^{9} \varphi^{3 / 2} F^{-1}\right) .
\end{aligned}
$$

С небольшим отклонением в значении коэффициентов это уравнение совпадает с рядом других уравнений, полученных в известных работах по полевой эмиссии (Элинсон [16], Модинос [17], Спиндт [18] и т. д.). Больше всего оно близко к уравнению Элинсона-Шредника, опубликованному в 1974 г. [16], поэтому формулу (4) можно назвать приближением Элинсона-Шредника в экспоненциальном виде.

Отметим, что все формулы в работе приведены с константами и переменными, выраженными в единицах СИ (за исключением работы выхода, выраженной в $\mathrm{eV})$, так что напряженность поля $F$ задается в $\mathrm{V} / \mathrm{m}$, а плотность тока $J-$ в $\mathrm{A} / \mathrm{m}^{2}$.

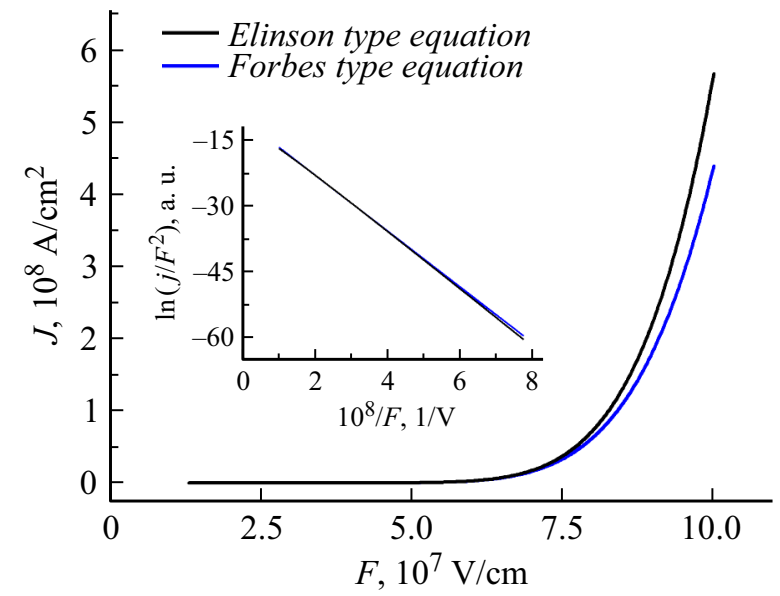

Рис. 1. Различие BAX в координатах Фаулера-Нордгейма для двух зависимостей: с использованием специальных функций Форбса и приближения Элинсона-Шредника при работе выхода $\varphi=4.6 \mathrm{eV}$.

Различие эмиссионных характеристик для зависимостей (3) и (4) показано на рис. 1 (графики представлены в удобном для восприятия виде: $F-$ в $\mathrm{V} / \mathrm{cm}$, а $J-$ в $\left.\mathrm{A} / \mathrm{cm}^{2}\right)$. При полях $10^{8} \mathrm{~V} / \mathrm{cm}$ различие плотностей тока достигает $10^{8} \mathrm{~A} / \mathrm{cm}^{2}$, что относительно много и должно существенно отражаться на вычислениях площади эмиссии при использовании неверной асимптотики.

\section{Варианты определения площади эмитирующей поверхности}

Рассмотрим простейший случай эмиссионной поверхности - идеальный полевой катод: эмиттер с однородным электрическим полем $F$ на поверхности, площадью $S_{0}$ и однородной работой выхода $\varphi$. Полный ток $I$ такого катода, согласно уравнению (1), формально можно записать, как

$$
I=S_{0} j_{\varphi}(F)=S_{0} A_{\varphi, F} F^{2} \exp \left(-B_{\varphi, F} / F\right),
$$

где $j_{\varphi}(F)$ - плотность тока эмиссии, определенная по одной из вышепредставленных формул, $S_{0}$ - площадь поверхности катода, $A_{\varphi, E}$ и $B_{\varphi}$, функции, зависящие от работы выхода материала $\varphi$ и слабо зависящие от величины электрического поля $F$.

Если же немного усложнить задачу и перейти к системе анод-катод, в которой анод по-прежнему считается плоским, а катод имеет произвольную форму поверхности, то применение формулы (5) станет невозможным. Вызывающее эмиссионный ток электрическое поле $F$ в разных точках поверхности катода будет определяться формой и материалом этой поверхности, а также взаимным расположением электродов. Для описания связи этого поля с макроскопическими параметрами системы 
вводят коэффициент усиления поля $\beta$ :

$$
\beta=F / F_{M}=F /(U / d),
$$

где $F_{M}$ - поле, которое получилось бы в идеальной плоскопараллельной системе (простой конденсатор), $U-$ приложенное к системе напряжение, $d-$ расстояние между анодом и катодом (в общем случае расстояние между их ближайшими точками).

Вместе с изменением однородности распределения поля на поверхности катода в расчетах его полного тока эмиссии возникает потребность интегрировать плотность тока $j$ по поверхности (область $K$ ):

$$
I=\int_{K} j(F) d S=\int_{K} A_{\varphi, F} F^{2} \exp \left(-B_{\varphi, F} / F\right) d S .
$$

Заметим, что в выражении (7) никакого параметра площади эмиссии в явном виде нет (кроме площади поверхности катода $K$, которая, между прочим, сама нуждается в отдельном определении). Таким образом, в общем случае площадь эмиссии не является частью фундаментального закона и требует своего строгого определения.

Обычно площадь эмиссии связывают с областью катода $K_{e}$ в окрестности точки с максимальной плотностью тока. В качестве критерия определения этой области можно взять „точечную“ характеристику: например, величину локального поля, необходимого для возникновения полевой эмиссии $\left(2 \cdot 10^{7} \mathrm{~V} / \mathrm{cm}\right.$ для работы выхода $4.5 \mathrm{eV}[16])$ или заданный минимальный уровень плотности тока (не менее $6 \mathrm{~A} / \mathrm{cm}^{2}[19,20]$ ). Также в качестве критерия можно взять интегральную характеристику - долю общего тока, которую должна давать $K_{e},\left(Q_{I}=I_{K e} / I=0.95\right)$ или уровень шума $\left(I_{\text {noise }} \sim 0.1 \mathrm{nA}\right)$, который должна давать вся остальная поверхность катода. Эти четыре критерия обеспечивают возможность аналитического расчета четырех различных видов площадей: $S_{E}, S_{j}, S_{\% l}, S_{\text {noise }}$.

Другим видом площади (с „интегрально-точечным“ критерием) является так называемая „notional emission area“ [21-23], которая вычисляется как отношение полного эмиссионного тока к характеристической плотности тока $j_{c}$ (максимальной плотности тока на вершине эмиттера):

$$
S_{\mathrm{Jtop}}=\frac{I}{j_{\max }}=\int_{K_{e}} \frac{j}{j_{\max }} d s .
$$

Эта условная площадь $S_{\text {Jtop }}$ отличается от представленных выше четырех отсутствием численного критерия, необходимого для ее расчета. Единственным условием является выбор области интегрирования $K_{e}$, которая из общих соображений должна представлять собой область катода, дающую эмиссионный ток.

В работе [24] было предложено еще одно определение площади эмиссии. Критерием ее нахождения является величина поля, которое возникало бы на вершине эмиттера при минимальном общем токе $(\sim 0.1 \mu \mathrm{A})$. Эта площадь $\left(S_{\text {Etop }}\right)$ характерна тем, что при потере эмиссионного сигнала экспериментатором ее величина автоматически становится равной нулю.

Среди экспериментаторов широко распространен метод определения площади эмиссии по анализу экспериментальной $\mathrm{BAX}[25,26]$. В этом случае реальному катоду сопоставляется некий идеальный эмиттер с площадью $S_{\text {eff }}$ и однородным полем $F_{\text {eff }}$ на поверхности, которому соответствует эффективный коэффициент усиления поля $\beta_{\text {eff. }}$ Эти эффективные параметры находятся из условия совпадения модельной ВАХ в координатах Фаулера-Нордгейма (ВАХ-ФН) этого идеального эмиттера с линией тренда, построенной для экспериментальной ВАХ-ФН методом наименьших квадратов. Зависимость тока от напряжения в модельной ВАХ

$$
I=S_{\mathrm{eff}} A_{\varphi, F_{\mathrm{eff}}} F_{\mathrm{eff}}^{2} \exp \left(-\frac{B_{\varphi, \mathrm{eff}}}{F_{\mathrm{eff}}}\right),
$$

где макроскопическое поле $F_{M}=U / d$, а поле $F_{\text {eff }}=F_{M} \beta_{\text {eff. }}$.

Если считать вариации работы выхода в области эмиссии незначительными, то для расчета коэффициентов $A_{\varphi, F_{\text {eff }}}$ и $B_{\varphi, F_{\text {eff }}}$ можно взять работу выхода, например, из литературных данных (4.6 eV для нанотрубок [27]).

Классический анализ ВАX основан на применении закона полевой эмиссии, в котором коэффициенты $A$ и $B$ не зависят от поля (приближение Элинсона). Он заключается в написании уравнения (9) в полулогарифмических координатах Фаулера-Нордгейма $X_{F}$ и $Y_{F}$, в которых модельная ВАХ становится прямолинейной:

$$
\begin{aligned}
Y_{F} & =\ln \left(\frac{A_{\varphi} S_{\text {eff-Elns }} \beta_{\text {eff-Elns }}^{2}}{d^{2}}\right)+\frac{B_{\varphi} d}{\beta_{\text {eff-Elns }}} X_{F} \\
& =\ln \left(R_{\mathrm{fit}}\right)+S_{\mathrm{fit}} X_{F},
\end{aligned}
$$

где $X_{F}=1 / U$ и $Y_{F}=\ln \left(I / U^{2}\right)$, а индекс Elns обозначает приближение Элинсона.

Экспериментальная ВАX строится в тех же координатах и аппроксимируется рямолинейной зависимостью, что позволяет найти наклон $S_{\text {fit }}$ и отсечку $\ln \left(R_{\mathrm{fit}}\right)$ для уравнения (10). По найденным величинам $R_{\mathrm{fit}}$ и $S_{\mathrm{fit}}$ вычисляются значения эффективных параметров $S_{\text {eff-Elns }}$ и $\beta_{\text {eff-Elns }}$ :

$$
\beta_{\mathrm{eff-E} \text {-lns }}=\frac{B_{\varphi} d}{S_{\mathrm{fit}}}, \quad S_{\mathrm{eff}-E l n s}=\frac{R_{\mathrm{fit}} d^{2}}{A_{\varphi} \beta_{\mathrm{eff}-E l n s}^{2}} .
$$

Практика показывает, что экспериментальные ВАХ-ФН зачастую существенно отличаются от прямой, что ставит под сомнение адекватность проводимой оценки площади $[28,29]$. К тому же теоретические расчеты показывают, что к искривлению ВАХ-ФН приводят эффекты взаимной экранировки эмиссионных центров [30], адсорбционно-десорбционные процессы [31], локальный джоулев разогрев [32], резкие перепады в распределении центров по величине коэффициента усиления по- 
ля [33,34], а также зависимость коэффициента усиления поля на поверхности эмиттера от межэлектродного расстояния [35].

С другой стороны, формально эффективную площадь эмиссии $S_{\text {eff }}$ можно считать еще одним определением площади эмиссии, которая требует построения ВАХ-ФН в заданном интервале напряжений и аппроксимации ее прямой линией. Именно интервал напряжений $\left(U_{0} \pm \Delta U\right)$ и будет являться критерием определения величины этой площади.

Искривление ВАХ-ФН может быть вызвано не только сопутствующими эффектами, но и отклонением самого закона эмиссии от прямой, которую задает выражение (10), так как в более точной записи закона (см. формулу (1)) коэффициенты $A_{\varphi, F}$ и $B_{\varphi, F}$ имеют свою зависимость от величины приложенного напряжения [36]. Учет этого искривления может быть сделан за счет изменения координат Фаулера-Нордгейма. Для этого в асимптотике Форбса (3) опускается слабая зависимость от поля функции $\tau_{F}$ (принимается $\tau_{F}^{2} \approx 1.1$ )

$$
I \approx S_{K} \frac{a_{F N} \varphi^{-1}}{1.1} \exp (\eta) F_{R}^{\eta / 6}\left(\beta F_{M}\right)^{2-\eta / 6} \exp \left(-\frac{B_{F N} \varphi^{3 / 2}}{\beta F_{M}}\right),
$$

где введена функция $\eta=b_{F N} \varphi^{3 / 2} / F_{R}$.

Введение новых координат Фаулера-Нордгейма: $X_{F}^{\prime}=1 / U$ и $Y_{F}^{\prime}=\ln \left(I / U^{k}\right)$, где $k=2-\eta / 6$, позволяет выпрямить модельную ВАХ-ФН и получить с помощью линейной аппроксимации экспериментальной ВАХ-ФН значения наклона $S_{\text {fit }}$ и отсечки $\ln \left(R_{\text {fit }}\right)$. Так же как и в случае приближения Элинсона, эти величины позволяют вычислить эффективные площадь эмиссии $S_{\text {eff-Frbs }}$ и коэффициент усиления поля $\beta_{\mathrm{eff}-F r b s}$ :

$$
\begin{gathered}
\beta_{\mathrm{eff}-F r b s}=-\frac{\varphi^{3 / 2} b_{F N} d}{S_{\mathrm{fit}}}, \\
S_{\mathrm{eff}-F r b s}=R_{\mathrm{fit}} \frac{\varphi \tau_{F N}^{2}}{a_{F N}} F_{R}^{-\eta / 6} e^{-\eta}\left(\frac{\beta_{\mathrm{eff}-F r b s}}{d}\right)^{-k},
\end{gathered}
$$

где величина $F_{R}$ - поле снятия потенциального барьера, зависящее от квадрата работы выхода (см. формулу (2)), $a_{F N}$ и $b_{F N}$ - константы Фаулера-Нордгейма, а индекс Frbs означает применение асимптотики Форбса.

Отметим, что существуют и другие методы учета более полной по сравнению с приближением Элинсона формулы полевой эмиссии при анализе ВАХ-ФН (например, в [37]), однако подходы эти справедливы только для идеального эмиттера, у которого коэффициент усиления поля одинаков для всей поверхности.

Описание полевых эмиттеров заданным законом полевой эмиссии позволяет получить еще один вид эффективной площади эмиссии. Способ основан на приравнивании производной тока $I$ по напряжению $U$ в заданной точке ВАХ к аналогичной производной идеального эмиттера с площадью $S_{\text {diff }}$ и усилением поля на поверхности $\beta_{\text {diff }}$ (математически это означает также равенство и производных, соответствующих ВАХ-ФН).
Производная тока по напряжению для закона полевой эмиссии с приближением Элинсона (см. формулу (4)) дает следующее выражение:

$$
\begin{aligned}
\frac{d I}{d U} & =\int_{K}\left(\frac{d j}{d U}\right) d S=\int_{K}\left(\frac{2 j}{U}-\frac{B_{\varphi} d}{U^{2} \beta} j\right) d S \\
& =\frac{2 I}{U}-\frac{B_{\varphi} d}{U^{2}} \int_{K}\left(\frac{j}{\beta}\right) d S=\frac{2 I}{U}-\frac{B_{\varphi} d}{U^{2}} \bar{I}_{\beta},
\end{aligned}
$$

где введена величина $\bar{I}_{\beta}-$ ток „усредненный по коэффициенту усиления поля“.

С другой стороны, модельная ВАХ (см. формулу (9)) имеет производную

$$
\frac{d I}{d U}=\frac{I}{U^{2}}\left(2 U-\frac{B_{\varphi} d}{\beta_{\mathrm{diff}}}\right)
$$

откуда

$$
\beta_{\mathrm{diff}}=\frac{I}{\bar{I}_{\beta}}
$$

Эффективная площадь находится из того же уравнения модельной ВАХ (см. формулу (11)) с использованием заданного уровня напряжения $U$ и величины эмиссионного тока $I$

$$
S_{\mathrm{diff}}=\frac{I}{U^{2} \beta_{\mathrm{diff}}^{2}} \frac{d^{2}}{A_{\varphi}} \exp \left(+\frac{B_{\varphi} d}{U \beta_{\mathrm{diff}}}\right)
$$

Получение эффективной площади для закона полевой эмиссии, основанном на приближении Форбса (см. формулу (3)), проводится аналогичным образом и дает следующие выражения для вычисления $\beta_{\text {diff }}$ и $S_{\text {diff: }}$

$$
\begin{gathered}
F_{U}\left(\beta_{\text {diff }}\right)=\frac{\overline{\bar{I}}_{\beta}}{I}, \\
S_{\text {diff }}=\frac{I}{a F_{R}^{2}} \frac{\tau\left(f_{\text {diff }}\right)^{2}}{f_{\text {diff }}^{2}} \exp \left(+\frac{b}{F_{R}} \frac{v\left(f_{\text {diff }}\right)}{f_{\text {diff }}}\right),
\end{gathered}
$$

где введены следующие функции:

$$
\begin{gathered}
\overline{\bar{I}}_{\beta}=\int_{K} j F_{U}(\beta) d S, \\
F_{U}(\beta)=2+\frac{b}{6 F_{R}} \frac{6-f_{\beta}}{f_{\beta}}+\frac{2 f_{\beta}\left(\ln f_{\beta}-1\right)}{18+2 f_{\beta}-f_{\beta} \ln f_{\beta}}, \\
f_{\beta}=\frac{U}{d F_{R}} \beta, \\
f_{\text {diff }}=\frac{U}{d F_{R}} \beta_{\text {diff. }}
\end{gathered}
$$

Сложность этих уравнений не позволяет решить их аналитически, поэтому в настоящей работе мы применили численные методы для нахождения $\beta_{\text {diff }}$ и $S_{\text {diff }}$ (поиск решения методом деления пополам). 


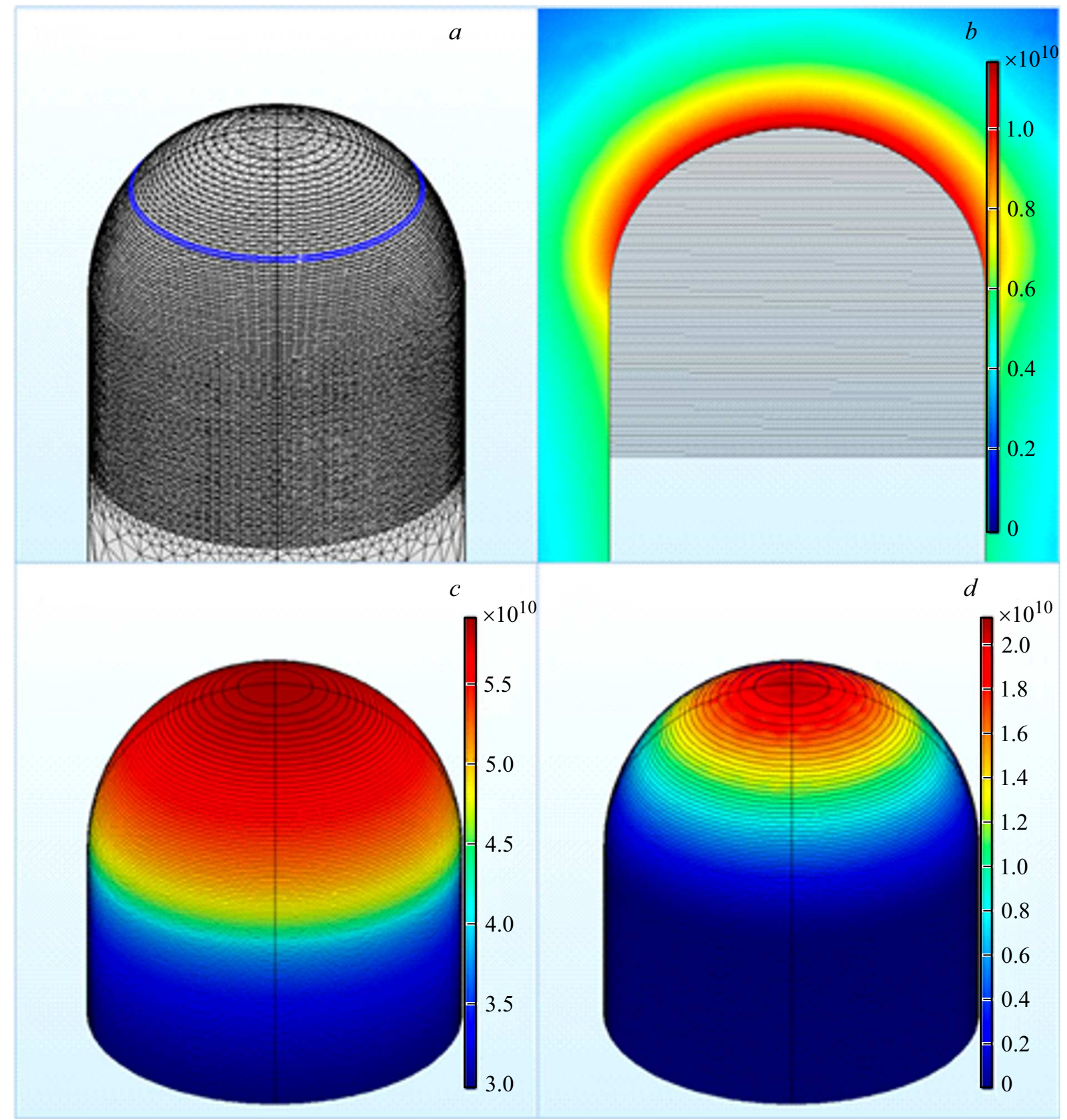

Рис. 2. Результаты расчета модели проводящей нанотрубки: $a-$ расчетная сетка вершины нанотрубки; $b-$ распределение напряженности электрического поля в межэлектродном пространстве $[\mathrm{V} / \mathrm{m}]$ при напряжении $U=4700 \mathrm{~V} ; c-$ распределение электрического поля поля $[\mathrm{V} / \mathrm{m}]$ при напряжении $U=2500 \mathrm{~V}$ и соответствующие ему распределение плотности тока $(d)$ по поверхности вершины $[\mathrm{A} / \mathrm{m} 2]$.

Исследование работы эмиттеров молекулярного масштаба (например, нанотрубок) сталкивается с трудностями описания процесса испускания электронов отдельными атомами [38]. Теоретические разработки предполагают использование метода функционалов и сложных квантово-механических расчетов [39]. Определение площади эмиссии для таких систем должно отличаться от приведенных выше определений. Площадь будет либо дискретной (если определить ее как число атомов, вероятность эмиссии электронов с которых превыша- ет заданный уровень), либо непрерывной (если эмиссионной поверхностью считать внешнюю поверхность электронных орбиталей молекул эмиттера). Наверняка возможны и другие виды „квантовой“ площади эмиссии, однако рассмотрение этих характеристик выходит за рамки настоящей работы.

В противовес сложности квантово-механических расчетов атомарного уровня существует метод определения площади эмиссии макроскопических эмиттеров по их геометрической форме $\left(S_{\mathrm{Geom}}\right)$. Например, в случае 
одноострийного эмиттера в виде вольфрамовой иглы, площадью эмиссии можно назвать площадь полусферы, вписанной в ее наконечник $\left(S_{\mathrm{Geom}}=2 \pi r^{2}\right.$, где $r$ радиус полусферы). Эту площадь часто применяют для численной оценки плотности эмиссионного тока на поверхности эмиттера по измеренному макроскопическому току. Критерием ее является способ оценки радиуса кривизны.

В случае острий сложной формы в качестве площади можно также принять площадь условного сечения $S_{\mathrm{Geom}}=\pi r^{2}$, где $r-$ средний радиус кривизны острия [20].

Отметим возможность расчета еще одного вида площади эмиссии $\left(S_{\beta}\right)$, которая определяется, как площадь катода, взвешенная по относительной величине электрического поля на его поверхности, и является аналогом условной площади $S_{\text {Jtop }}$ (площадь катода, взвешенная по плотности тока):

$$
S_{\beta}=\int_{K} \frac{E}{E_{\max }} d s=\frac{1}{\beta_{\max }} \int_{K} \beta d s .
$$

Отношение этой площади к площади катода показывает, во сколько раз средний формфактор меньше максимального. Так же как и геометрическая площадь $S_{\mathrm{Geom}}$, эта площадь абсолютно не зависит от величины приложенного к эмиттеру напряжения и определяется только его формой, а критерием ее определения служит граница области катода, по которой производится интегрирование.

\section{Моделирование, расчет и сравнение видов эмиссионных площадей}

Методом компьютерного моделирования в среде COMSOL Multyphysics 5.3 нами была построена модель полевого катода в виде проводящего острия полусфера на цилиндре в плоскопараллельной системе электродов (рис. 2,a).

Параметры системы: диаметр пластин электродов $D_{a}=200 \mu \mathrm{m}$, высота острия катода $H=5 \mu \mathrm{m}$, диаметр острия $D_{o}=8 \mathrm{~nm}$, вершина острия - полусфера с радиусом $r=4 \mathrm{~nm}$, расстояние между концом острия и анодом $D_{\text {aо }}=300 \mu \mathrm{m}$.

За основу параметров проводящего острия были взяты геометрические параметры нанотрубок, которые мы используем в эксперименте (Таунит-М, Тамбов: многостенные углеродные нанотрубки диаметром 8-10 nm, длиной не менее $2 \mu \mathrm{m})$.

Пластины электродов в эксперименте имеют диаметр $10 \mathrm{~mm}$, что намного превышает размеры электродов в модели. Для устранения ожидаемых краевых эффектов мы поставили условие, что поля на границах виртуального цилиндра не искривляются (так называемое граничное условие Неймана).

Расчет полей (рис. 2, $b, c$ ) показал, что при напряжении $U=2.5 \mathrm{kV}$ напряженность поля на вершине острия

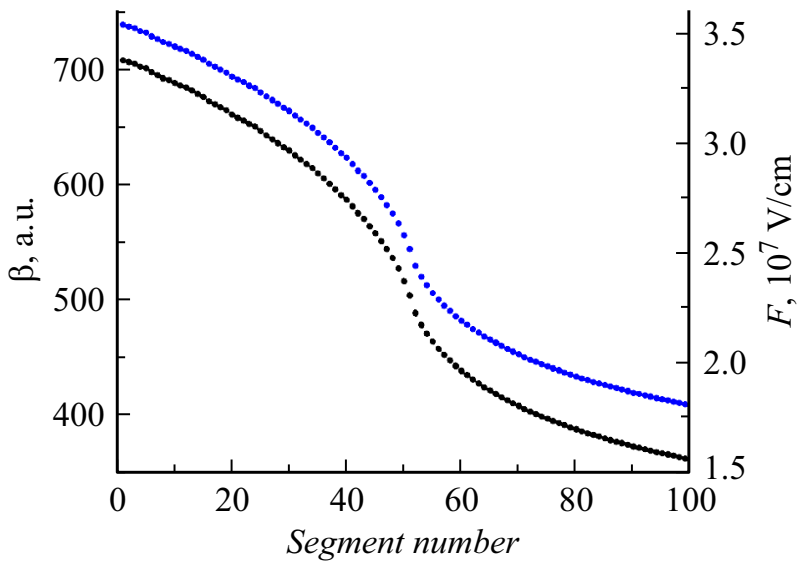

Рис. 3. Зависимость коэффициента усиления (нижняя кривая) и напряженности электрического поля (верхняя кривая) от номера сегмента на вершине острия УНТ (сплошная линия сплайн).

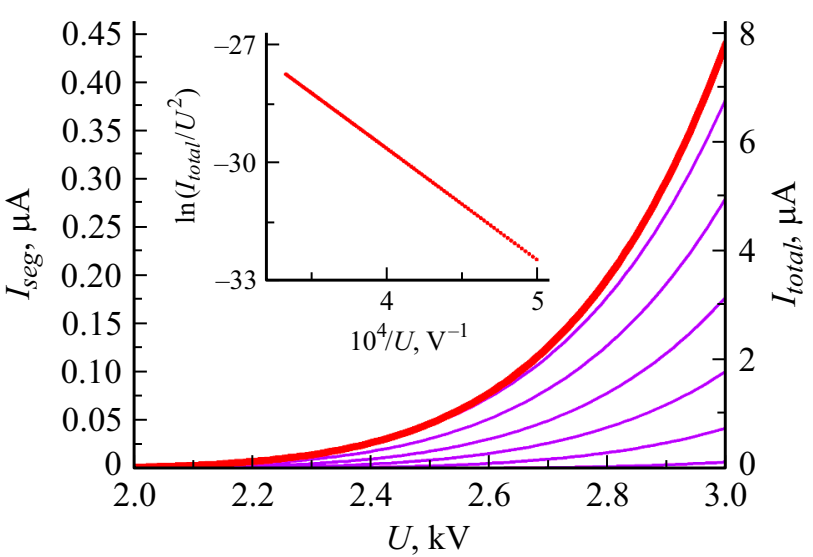

Рис. 4. ВАX каждого десятого сегмента модели (тонкие линии) и ВАХ всего эмиттера (толстая линия). На вставке: ВAX- $\Phi$ H.

равна $E_{0}=5.89 \cdot 10^{7} \mathrm{~V} / \mathrm{cm}$. Этот результат согласуется с литературными данными по моделированию аналогичных систем в других программных средах $[10,26]$.

Для получения общей $\mathrm{BAX}$ модельного эмиттера его вершина была виртуально разделена на сегменты равной площади: пятьдесят сегментов в полусфере и еще пятьдесят на цилиндре под ней (рис. 2,d). Площадь вершины-полусферы составила $100.5 \mathrm{~nm}^{2}$, а высота одного сегмента $-0.08 \mathrm{~nm}$.

В каждом сегменте было вычислено электрическое поле у его верхнего края при напряжении $1.5 \mathrm{kV}$ и найден соответствующий коэффициент усиления поля $\beta$ (см. формулу (6)). При этом за исходное поле $E_{M}$ бралось поле возле поверхности анода. Полученный профиль эмиссионной системы (рис. 3) был детализирован сплайн-интерполяцией (число сегментов увеличено до 1000) и использовался для дальнейших расчетов в среде графического программирования LabView 2016. 

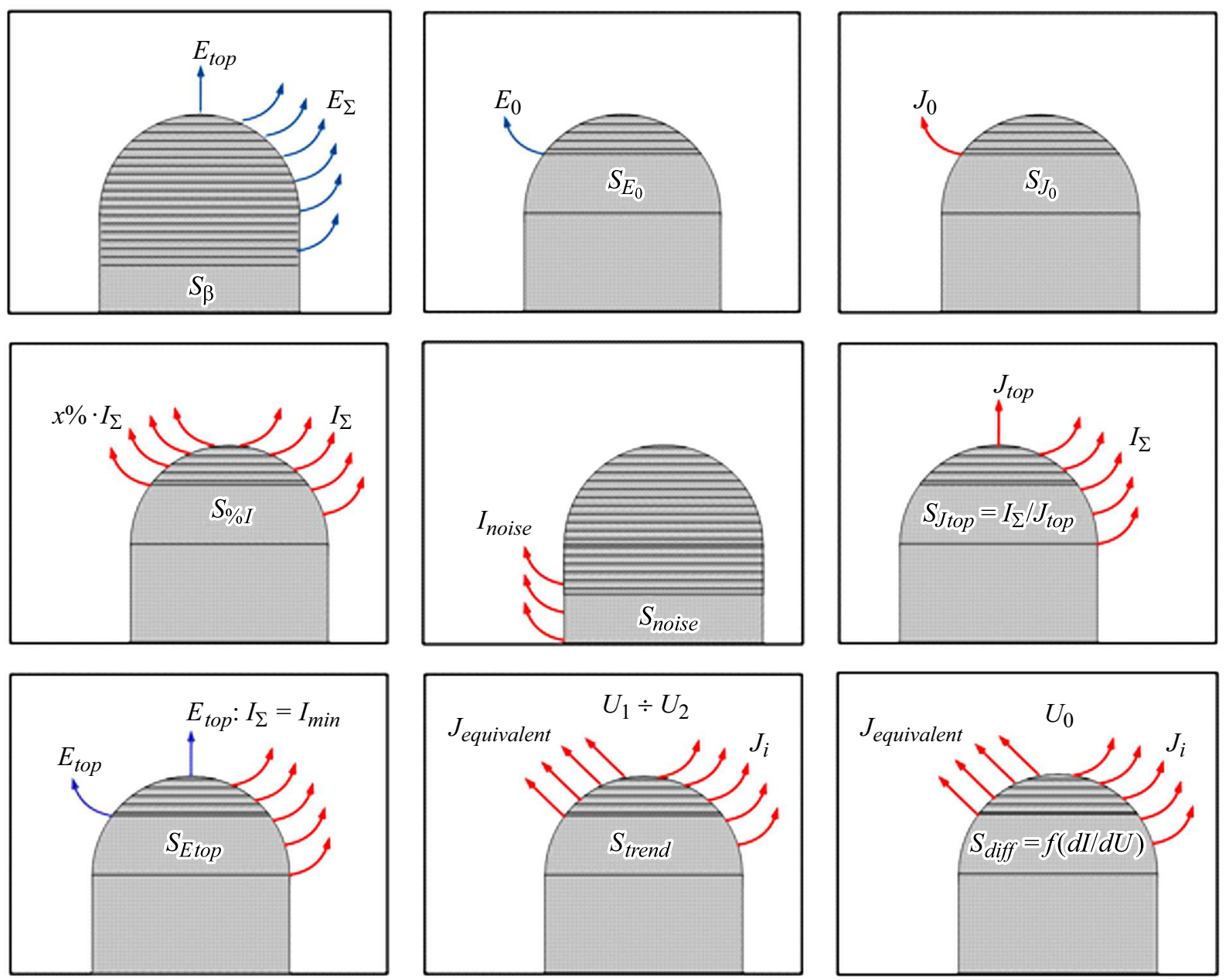

Рис. 5. Схематическое изображение площадей эмиссии предложенной модели: геометрической $-S_{\text {Geom}}$, средней взвешенной по величине поля $-S_{\beta}$, найденной по локальному полю $-S_{E}$, найденной по локальной плотности тока $-S_{j}$, заданной процентом суммарного тока $-S_{\% l}$, заданной уровнем шума $-S_{\text {noise }}$, найденной по плотности тока на вершине $-S_{\text {Jtop, }}$ заданной минимальным уровнем тока $-S_{\text {Etop }}$, эффективной для ВАХ-ФН $-S_{\text {eff }}$ и дифференциальной для ВАХ $-S_{\text {diff. }}$

Отметим, что используемые в модели размеры сегментов формально меньше атомарных, однако проводимое разбиение не влияет на расчет электростатических полей, так как в используемом программном пакете размерности носят лишь относительный характер. Очевидно, что для многостенных нанотрубок достаточно большого диаметра мы можем рассматривать поверхность как атомарно гладкую.

Пренебрегая токоотдачей поверхности эмиттера, расположенной за пределами разбитой на сегменты вершины, полный эмиссионный ток можно считать суммой токов 1000 сегментов модели (похожий подход к расчету величины эмиссионного тока был применен в работе [40]). Принимая плотность тока каждого сегмента примерно равной плотности тока у его верхнего края $J_{i}$, суммарный ток эмиттера можно вычислить по формуле

$$
I=S_{s} \sum_{n} j_{n}=S_{s} \sum_{n} A\left(U, \beta_{n}\right)\left(U \beta_{n} / d\right)^{2} e^{-B\left(U, \beta_{n}\right) d /\left(U \beta_{n}\right)},
$$

где $S_{s}=2 \pi h r$ - площадь каждого сегмента модельного эмиттера (для полусферы и цилиндра площади сегментов равной высотой равны), $r$ - радиус сферы на конце острия, $h-$ высота сегментов $(h=r / 50)$, $U$ - приложенное к системе напряжение (в вольтах), $d$ - расстояние между концом острия и анодом, $\beta-$ формфактор, не зависящий от $d$, а функции $A$ и $B$ определяются законом полевой эмиссии.

В настоящей работе мы использовали асимптотику Форбса (3) с работой выхода материала $\varphi=4.6 \mathrm{eV}$ [27]. На рис. 4 представлены ВАХ отдельных сегментов, а также общая BAX эмиттера в диапазоне напряжений от 2 до $3 \mathrm{kV}$ (этот диапазон удовлетворяет требованиям теста Форбса на вершине эмиттера, где $\beta \approx 700$ [15]).

Коэффициенты усиления поля сегментов, BAX каждого сегмента и общая $\mathrm{BAX}$ всего эмиттера позволили провести оценку десяти видов площади эмиссии, которые были описаны в предыдущем разделе. Расчет каждой площади производился в отдельной подпрограмме, выполненной на языке LabView. На рис. 5 представлено 
Результаты оценки площади эмиссии смоделированной нанотрубки различными точечными и интегральными методами для $U=1.5 \mathrm{kV}$

\begin{tabular}{c|c|c|c|c}
\hline Вид площади & Критерий вычисления & $\begin{array}{c}\text { Величина в } \mathrm{nm}^{2} \\
\text { при } U=1.5 \mathrm{kV}\end{array}$ & $\begin{array}{c}\text { Число } \\
\text { сегментов }\end{array}$ & $\begin{array}{c}\text { Критерий } \\
\text { для 130 сегментов }\end{array}$ \\
\hline$S_{\text {Geom }}$ & полусфера & 100.53 & 500 & - \\
$S_{\beta}$ & границы модели & 147.73 & 147 & - \\
$S_{E}$ & $E_{\min }=2 \cdot 10^{7} \mathrm{~V} / \mathrm{cm}$ & 146.83 & 731 & $E_{\min }=3.4 \cdot 10^{7} \mathrm{~V} / \mathrm{cm}^{2}$ \\
$S_{j}$ & $J_{\min }=6 \mathrm{~A} / \mathrm{cm}^{2}$ & 131.97 & 657 & $J_{\min }=2.5 \cdot 10^{6} \mathrm{~A} / \mathrm{cm}^{2}$ \\
$S_{\% I}$ & $Q_{I}=95 \%$ & 55.04 & 274 & $Q_{I}=69 \%$ \\
$S_{\text {noise }}$ & $I_{\text {nоise }}=0.1 \mathrm{nA}$ & 10.65 & 53 & $I_{\text {noise }}=4.8 \cdot 10^{-11} \mathrm{~A}$ \\
$S_{\text {Jtop }}$ & границы модели & 26.17 & 0 & $\equiv$ \\
$S_{\text {Etop }}$ & $I_{\text {thresh }}=0.1 \mu \mathrm{A}$ & 0 & 334 & $I_{\text {thresh }}=6.4 \cdot 10^{-11} \mathrm{~A}$ \\
$S_{\text {eff-Elns }}$ & $2 \Delta U=1000 \mathrm{~V}$ & 67.24 & 252 & - \\
$S_{\text {eff-Frbs }}$ & & 50.67 & 279 & - \\
$S_{\text {diff-Elns }}$ & число сегментов & 56.22 & 274 & -
\end{tabular}

схематическое изображение искомых площадей и методов их вычисления.

В таблице представлены результаты оценок десяти видов площадей эмиссии (последние две разбиваются на приближения Элинсона и Форбса), которые были получены для напряжения $1.5 \mathrm{kV}$ (это напряжение, при котором наблюдается полевая эмиссия для макроскопических нанокомпозитных эмиттеров на основе нанотрубок [41]). В отдельном столбце указано число сегментов, которое охватывает каждая рассчитанная площадь на вершине модели (всего сегментов в модели 1000).

Вариация величин критериев площадей $\left(E_{\min }, J_{\min }, Q_{I}\right.$, $\left.I_{\text {thresh }}, I_{\text {noise }}\right)$ позволила совместить соответствующие площади в одной точке при напряжении $1.5 \mathrm{kV}$. За базовую величину площади при этом была взята величина площади $S_{\text {Etop }}$, которая не имеет численного критерия в своем определении (число сегментов $N=130$ ). Величины критериев, необходимые для этого совмещения, представлены в последнем столбце таблицы.

На рис. 6, $a$ представлена зависимость исследуемых видов площадей от уровня напряжения $U$ в теоретически допустимом диапазоне от 1 до $6.3 \mathrm{kV}$ (при $U<1 \mathrm{kV}$ поле на вершине эмиттера меньше $10^{7} \mathrm{~V} / \mathrm{cm}$, что гарантирует отсутствие эмиссии, а при $U>6.3 \mathrm{kV}$ теоретические оценки дают падение потенциального барьера на границе эмиттер-вакуум).

На рис. $6, b$ показана зависимость от напряжения доли тока, соответствующей рассчитанным площадям $\left(I_{S} / I\right.$, где $I_{S}$ - ток области $K_{e}$, состоящей из сегментов модели, суммарная площадь которых равна соответствующей площади эмиссии).

Полученные кривые демонстрируют принципиально различный характер. Площади $S_{\mathrm{Geom}}$ и $S_{\beta}$ являются константами, не зависящими от напряжения, но не являются асимптотами ни для одной из других площадей. Примечательно, что доля тока в площади $S_{\beta}$ близка к $100 \%$ во всем диапазоне, а в $S_{\mathrm{Geom}}$ постепенно падает от 100 до 96\%, т.е. 4\% тока переходит из нее на цилиндрическую часть катода.

Площади $S_{E}$ и $S_{j}$ имеют идентичную зависимость от поля (в силу связи их критериев через уравнение полевой эмиссии: каждому выбранному $E_{\min }$ соответствует свой $\left.J_{\min }\right)$, однако достаточно быстро охватывают все сектора модели, так что доля их токового вклада почти сразу становится $100 \%$.

Площади $S_{\text {noise }}$ и $S_{\text {Etop }}$ и их токовый вклад также имеют достаточно крутой характер, приводящий их к насыщению при 3 и $4 \mathrm{kV}$ соответственно. Примечательно, что зависимость $S_{\text {noise }}$ обладает необычной волнистой формой, что, возможно, связано с ограниченностью области интегрирования нижней части катода.

Площади $S_{\% I}$ и $S_{\text {Jtop }}$ демонстрируют постоянно растущий, асимптотический характер, что связано с привязанностью их критериев к полному эмиссионному току. Однако если токовый вклад площади $S_{\% I}$ является константой (в силу ее определения), то площадь $S_{\text {Jtop }}$ дает от 68 до 75\% общего тока.

Аппроксимационная площадь $S_{\text {eff-Frbs }}$ оказалась близка к $S_{\% I}$, но с ростом напряжения постепенно отстает от нее, что сказывается на ее токовом вкладе, который падает с 95 до 91\%. Однако при этом $S_{\text {eff-Frbs }}$ постепенно увеличивает разницу с условной площадью $S_{\text {Jtop }}$, опережая ее на величину от 25 до $35 \mathrm{~nm}^{2}$.

Дифференциальная площадь $S_{\text {diff-Frbs }}$ оказалась выше аппроксимационной $S_{\text {eff-Frbs }}$, что связано с учетом в ней зависимости $\tau(F)$ (отметим, что замена $\tau$ в исходной формуле на константу 1.1 приводит к равенству кривых). Более того, токовый вклад этой площади постепенно растет с 95 до 97\%.

Неожиданным результатом оказалось то, что площадь

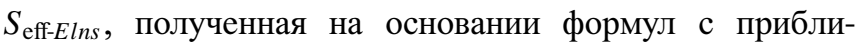
жением Элинсона (напомним, что эмиссионные токи в модели были рассчитаны по закону с асимптотикой Форбса (3)), с ростом уровня напряжения падает, причем примерно с той же скоростью, что растет ее аналог 

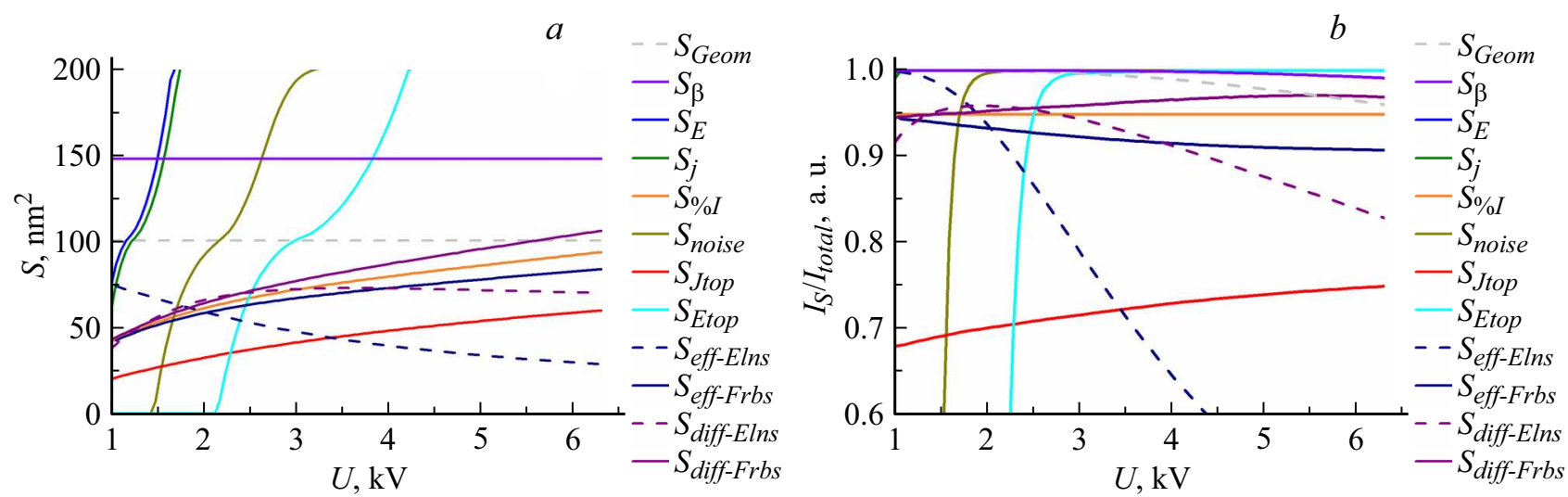

Рис. 6. Зависимости от напряжения величин эмиссионных площадей различных видов $(a)$ и доля от общего тока, которую они эмитируют $(b /)$. Зависимость от $U$ аппроксимационных площадей $S_{\text {eff-Elns }}$ и $S_{\text {eff-Frbs }}$ строилась для скользящего диапазона $U \pm \Delta U$, где $2 \Delta U=100 \mathrm{~V}$.
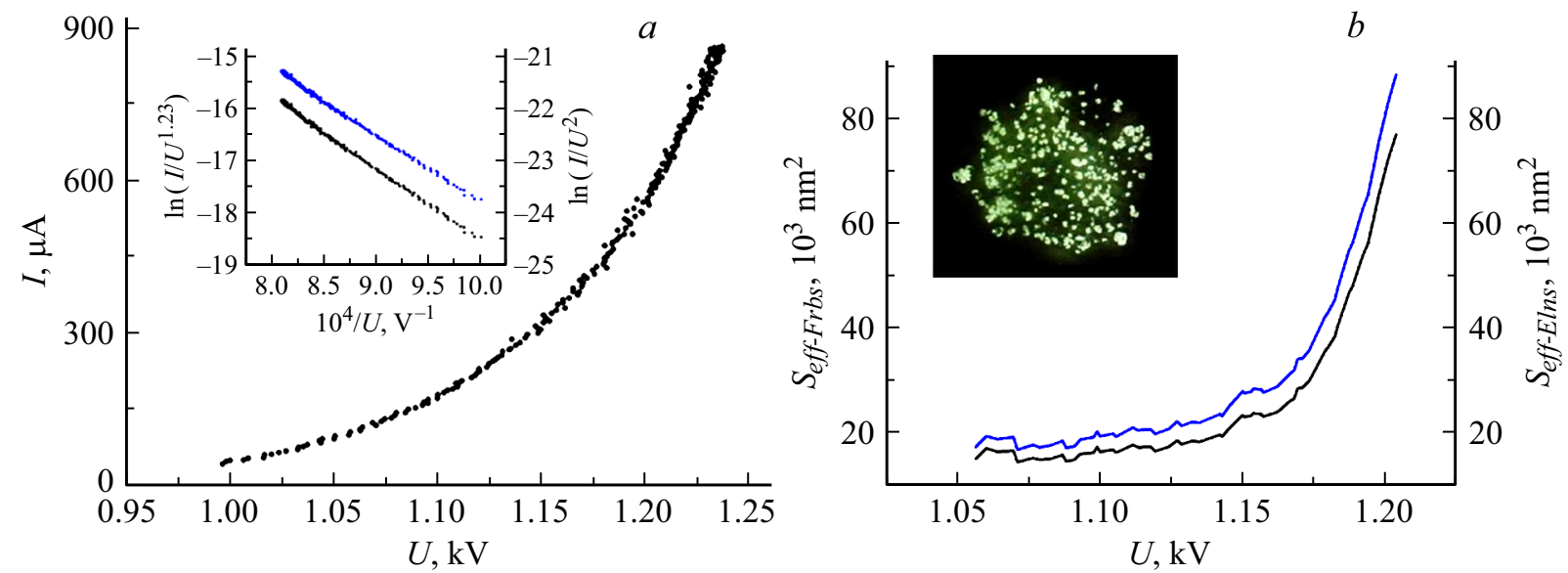

Рис. 7. Экспериментальные данные: $a-$ полная ВАХ полевого катода (на вставке, ВАХ-ФН с координатами $Y=\ln \left(I / U^{2}\right)$ и $\left.Y=\ln \left(I / U^{1.23}\right)\right), b$ - зависимость эффективной площади эмиссии от середины диапазона аппроксимации шириной $2 \Delta U=100 \mathrm{~V}$, полученная для двух теоретических приближений Элинсона и Форбса (на вставке свечение эмиссионных центров, полученное полевым эмиссионным проектором).

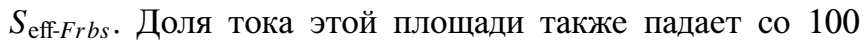
до $43 \%$. Анализ ВАХ-ФН с различной степенью $\mathrm{k}$ показал, что эффект этот связан с направлением ее изгиба: при изменении степени в координате $\mathrm{Y}$ выпуклость кривой меняется на противоположную (максимальная прямолинейность ВАХ-ФН достигается при $k=1.54)$.

Последняя площадь $S_{\text {diff-Elns }}$ также продемонстрировала нестандартное поведение: небольшое опережение площади $S_{\text {diff-Elns }}$ в начале и плавное падение в конце, что также указывает на ошибочность применения формул, основанных на приближении Элинсона.

\section{Экспериментальное определение площади эмиссии}

Приведем пример получения площади эмиссии $S_{\text {eff }}$ методом анализа экспериментального ВАХ-ФН. В качестве исследуемого образца нами был взят много- острийный полевой эмиттер на основе нанокомпозита „полимер/углеродные нанотрубки“ (Таунит-М, Тамбов). Поверхность таких катодов далека от идеальной и обладает своим распределением коэффициентов усиления поля по поверхности. Результат такой неоднородности сказывается на прямолинейности ВАХ-ФН, так что она изгибается гораздо сильнее, чем ВАХ-ФН смоделированной нанотрубки [34].

На рис. 7, $a$ представлена экспериментальная ВАХ, полученная с помощью уникальной компьютеризированной методики [42], а также ВАХ-ФН, построенная с применением двух различных координат $Y=\ln \left(I / U^{2}\right)$ и $Y=\ln \left(I / U^{1.23}\right)$ для двух теоретических приближений Элинсона и Форбса.

На рис. 7, $b$ представлены результаты расчета эффективной площади эмиссии $S_{\text {eff }}$ по линии тренда для ВАХ-ФН. Зависимости вычисленных площадей $\left(S_{\text {eff-Elns }}\right.$ и $\left.S_{\text {eff- } F r b s}\right)$ близки по форме, что говорит о том, что соответствующие ВАХ-ФН имеют одинаковую выпуклость. 
Отсутствие сильной зависимости площади от выбора приближения указывает на сильное отличие распределения коэффициента усиления поля по поверхности исследованного катода от рассмотренной выше модели единичной нанотрубки.

\section{Заключение}

Поднят вопрос о сущности параметра „площадь полевой эмиссии“ для протяженных эмиссионных структур. Представленная работа не является обзорной, однако включает в себя наиболее распространенные подходы к вычислению этого параметра и направлена на строгую формализацию этой величины. Рассмотрены десять различных видов определения площади с применением точечных, интегральных, дифференциальных и аппроксимационных критериев.

Для анализа свойств и соотношения площадей была использована модель одноострийного полевого катода с параметрами проводящей углеродной нанотрубки, расположенной в плоскопараллельной системе электродов. Модель была построена в программном пакете COMSOL Multiphysics, который позволил рассчитать распределение полей на поверхности вершины эмиттера. Расчеты плотности тока были произведены с применением уравнения Мерфи-Гуда в современной аннотации Форбса. На основе модельных данных были произведены оценки представленных в работе видов площади эмиссии и построены их зависимости от уровня напряжения $S(U)$, а также зависимости соответствующей им доли эмиссионного тока. По характеру зависимостей площади можно разделить на четыре группы:

1) не зависящие от напряжения и определяемые областью интегрирования $S_{\mathrm{Geom}}$ и $S_{\beta}$;

2) имеющие резкую зависимость и сводимые друг к другу вариацией численных критериев $S_{E}, S_{j}, S_{\text {noise, }}$ $S_{\text {Etop; }}$

3) плавно растущие, но имеющие принципиально различные доли тока $S_{\%}, S_{\text {Jtop }}, S_{\text {eff-Fr } b s}, S_{\text {diff-Frbs }}$;

4) имеющие падающую зависимость и требующие введения поправочных функций $S_{\text {eff-Elns }}$ и $S_{\text {diff-Elns }}$.

Показано, что наиболее часто используемая теоретиками условная площадь эмиссии $\left(S_{\mathrm{Jtop}}\right)$, рассчитанная для построенной в работе модели, включает в себя всего $68-75 \%$ эмиссионного тока, тогда как эффективная площадь эмиссии $\left(S_{\text {eff-Elns }}\right)$ заключает в себе $100-43 \%$ эмиссионного тока, однако с ростом напряжения ее величина и доля тока падают.

Для более корректного расчета эффективной площади рекомендуется применять модифицированные координаты Фаулера-Нордгейма (с напряжением в степени $k=1.23$ ), учитывающие особенности закона полевой эмиссии, или вводить дополнительные поправочные функции.

Некоторые определения площади $\left(S_{\mathrm{diff}}, S_{\mathrm{eff}-F}\right.$ и $\left.S_{\beta}\right)$ были предложены авторами по аналогии с уже суще- ствующими теоретическими подходами. Возможно, эти площади появляются в литературе впервые, поэтому их свойства представляют собой отдельный интерес.

Дифференциальная $S_{\text {diff }}$ является идеализированной теоретической площадью. Она требует знания закона полевой эмиссии и в ее расчетах используются достаточно сложные функции, включая интегрирование плотности тока по поверхности катода. Ее величина с ростом напряжения плавно растет, как и доля тока, которая имеет достаточно высокие значения 95-97\%.

Площадь $S_{\text {eff- } F r b s}$ может быть широко использована экспериментаторами. Как показывают экспериментальные данные, ее величина на практике лишь немного ниже стандартной площади $S_{\text {eff-Elns }}$, а теоретически ее применение более оправдано.

Площадь $S_{\beta}$ обладает наиболее стабильными характеристиками и конкурирует с простейшей $S_{\mathrm{Geom}}$, не основываясь на интуитивном выборе зоны эмиссии, но используя интегрирование поля по поверхности катода.

\section{Конфликт интересов}

Авторы заявляют, что у них нет конфликта интересов.

\section{Список литературы}

[1] Fursey G.N. Field Emission in Vacuum Microelectronics. NY.: Kluwer Academic, 2005. 205 p.

[2] Egorov N.V., Sheshin E.P. Field Emission Electronics. Springer Series in Advanced Microelectronics. Vol. 60. 2017. $568 \mathrm{p}$.

[3] Evtukh A., Hartnagel H., Yilmazoglu O., Mimura H., Pavlidis D. Vacuum Nanoelectronic Devices: Novel Electron Sources and Applications. John Wiley \& Sons, Ltd. West Sussex, UK, 2015. 464 p.

[4] Parmee R.J., Collins C.M., Milne W.I., Cole M.T. // Nano Convergence. 2015. Vol. 2:1. P. 1-27.

[5] Chubenko O., Baturin S.S., Kovi K.K., Sumant A.V., Baryshev S.V. // ACS. Appl. Mater. Interfaces. 2017. Vol. 9. N 38. P. 33229-33237.

[6] Patra R., Singh A., Vankar V.D., Ghosh S. // Adv. Mater. Lett. 2016. Vol. 7. N 10. P. 771-776.

[7] Navitski A. Scanning field emission investigations of structured CNT and MNW cathodes, niobium surfaces and photocathodes. Doctoral dissertation. University of Wuppertal, Germany, 2010. 133 p. URN (NBN): 46820101222-111353-7.

[8] Lysenkov D., Abbas H., Müller G., Engstler J., Budna K.P., Schneider J.J. // J. Vac. Sci. Technol. B. 2005. Vol. 23. N 2. P. 809-813.

[9] Kim D., Bourée J.-E., Kim. S.Y. // J. Appl. Phys. 2009. Vol. 105. P. 084315.

[10] Roveri D.S., Sant'Anna G.M., Bertan H.H., Mologni J.F., Alves M.A.R., Braga E.S. // Ultramicroscopy. 2016. Vol. 160. P. $247-251$.

[11] Fowler R.H., Nordheim L.W. // Proc. R. Soc. London Ser. A. 1928. Vol. 119. N 781. P. 173.

[12] Nordheim L.W. // Proc. R. Soc. London Ser. A. 1928. Vol. 121. N 788. P. 626. 
[13] Murphy E.L., Good R.H. // Phys. Rev. 1956. Vol. 102. P. 1464 1473.

[14] Forbes R.G., Deane J.H.B. // Proc. R. Soc. A. 2007. Vol. 463. P. 2907-2927.

[15] Forbes R.G. // Proc. R. Soc. A. 2013. Vol. 469. P. 20130271.

[16] Елинсон М.И. Ненакаливаемые катоды. М.: Сов. радио, 1974. $336 \mathrm{c}$.

[17] Модинос А. Авто-, термо- и вторично-электронная эмиссионная спектроскопия. М.: Наука, Гл. ред. Физматлит, 1990. $320 \mathrm{c}$.

[18] Spindt C.A., Brodie I., Humphrey L., Westerberg E.R. // J. Appl. Phys. 1976. Vol. 47. P. 5248-5263.

[19] Никиборов К.А., Егоров Н.В. Вестн. СПб. Ун-та. 2006. Cер. 10. Вып. 2. Прикладная математика, информатика, процессы управления. С. 39-45.

[20] Dyke W.P., Trolan J.K. // Phys. Rev. 1953. Vol. 89. P. 799.

[21] Podenok S., Sveningsson M., Hansen K., Campbell E.E.B. // NANO: Brief Reports and Reviews. 2006. Vol. 1. N 1. P. 8793.

[22] Forbes R.G., Deane J.H.B., Fischerc A., Mousad M.S. // Jordan J. Phys. 2015. Vol. 8. N 3. P. 125-147.

[23] Tang W.W., Shiffler D.A., Harris J.R., Jensen K.L., Golby K., LaCour M., Knowles T. // AIP Advances. 2016. Vol. 6. N 095007. P. 095007-1-9.

[24] Автореф. канд. дис. Бурцев А.А. Матричные автоэмиссионные катоды из монолитных углеродных материалов для приборов вакуумной электроники: Саратов, Россия, 2011. $126 \mathrm{c}$.

[25] Liu H., Kato S., Saito Y. // Nanotechnology. 2009. Vol. 20. P. 275206

[26] Berdinsky A.S., Shaporin A.V., Yoo J.-B., Park J.-H., Alegaonkar P.S., Han J.-H., Son G.-H. // Appl. Phys. A. 2006. Vol. 83. P. 377-383.

[27] Gao R., Pan Z., Wang Z. // Appl. Phys. Lett. 2001. Vol. 78. P. 1757.

[28] Zhu C., Lou C., Lei W., Zhang X. // Appl. Surf. Sci. 2005. Vol. 251. P. 249-253.

[29] Aplina K.L., Kent B.J., Song W., Castelli C. // Acta Astronautica. 2009. Vol. 64. P. 875-881.

[30] Eletskii A.V., Bocharov G.S. // Plasma Sources Sci. Technol. 2009. Vol. 18. N 034013. P. 1-8.

[31] Chen J., Li J., Yang J., Yan X., Tay B.K., Xue Q. // Appl. Phys. Lett. 2011. Vol. 99. P. 2011-2014.

[32] Cahay M., Zhu W., Fairchild S., Murray P., Back T., Gruen G. // Appl. Phys. Lett. 2016. Vol. 108. P. 0333110/5.

[33] De Assis T.A., Dall'Agnol F.F., Andrade R.F.S. // J. Phys. D: Appl. Phys. 2016. Vol. 49. P. 355301/11.

[34] Popov E.O., Kolosko A.G., Filippov S.V. // IEEE 30th Int. Vac. Nanoelectr. Conf. Herzogssaal Regensburg, Germany, 2017. P. 50-51.

[35] Thomas C.T., Michaels Th., Cabrera H., Zanin D.A., De Pietro L., Ramsperger U., Vindigni A., Pescia D. // Proc. Math. Phys. Eng. Sci. 2014. Vol. 470. N 2167. P. 20140014-126.

[36] Forbes R.G. // J. Vac. Sci. Technol. B. 1999. Vol. 17. N 2. P. 526-533.

[37] Forbes R.G. // IEEE 28th Int. Vac. Nanoelectr. Conf. Guangzhou, China, 2015. P. 70.

[38] Saito Y., Hataa K., Takakura A., Yotani J., Uemura S. // Physica B: Condens. Matter. 2002. Vol. 323. N 1-4. P. 30-37.

[39] Sorokina V., Nikiforov K. // Young Researchers in Vacuum Micro/Nano Electronics (VMNE-YR), Saint-Petersburg, Russia. 2016. P. 84-87.
[40] Ravi M., Bhat K.S., Khaneja M., Chaudhury P.K., Harsh A. // IEEE Intern. Vacuum Electron. Conf. (IVEC), Bangalore, Karnataka, India, 2011. P. 189-190.

[41] Popov E.O., Kolosko A.G., Filippov S.V., Romanov P.A., Terukov E.I., Shchegolkov A.V., Tkachev A.G. // Appl. Surf. Sci. 2017. Vol. 424. P. 239-244.

[42] Попов Е.О., Колосько А.Г., Филиппов С.В., Романов П.А., Федичкин И.Л. // Наноматериалы и наноструктуры - XXI век. 2016. Т. 7. № 1. С. 14-26. 